\title{
ON A $p$-LAPLACIAN TYPE OF EVOLUTION SYSTEM AND APPLICATIONS TO THE BEAN MODEL IN THE TYPE-II SUPERCONDUCTIVITY THEORY
}

\author{
By \\ HONG-MING YIN \\ Department of Mathematics, University of Notre Dame, Notre Dame, IN \\ Dedicated to Professor John R. Cannon on the occasion of his 60th birthday
}

\begin{abstract}
In this paper we study the Cauchy problem for a $p$-Laplacian type of evolution system $\mathbf{H}_{t}+\nabla \times\left[|\nabla \times \mathbf{H}|^{p-2} \nabla \times \mathbf{H}\right]=\mathbf{F}$. This system governs the evolution of a magnetic field $\mathbf{H}$, where the displacement currently is neglected and the electrical resistivity is assumed to be some power of the current density. The existence, uniqueness, and regularity of solutions to the system are established. Furthermore, it is shown that the limit solution as the power $p \rightarrow \infty$ solves the problem of Bean's model in the type-II superconductivity theory. The result provides us information about how the superconductor material under the external force becomes the normal conductor and vice versa. It also provides an effective method for finding numerical solutions to Bean's model.
\end{abstract}

1. Introduction. In this paper we shall study the following degenerate evolution system:

$$
\begin{gathered}
\mathbf{H}_{t}+\nabla \times\left[|\nabla \times \mathbf{H}|^{p-2} \nabla \times \mathbf{H}\right]=\mathbf{F}, \quad(x, t) \in Q_{T}, \\
\nabla \cdot \mathbf{H}=0, \quad(x, t) \in Q_{T}, \\
\mathbf{H}(x, 0)=\mathbf{H}_{0}(x), \quad x \in R^{3},
\end{gathered}
$$

where $Q_{T}=R^{3} \times(0, T]$ for some $T>0$ and $p>2$. Here a bold letter represents a vector in $R^{3}$.

The system (1.1) is derived from Maxwell's system where the current displacement is neglected since it is small in comparison with eddy currents (see Landau and Lifschitz [12]). The vector $\mathbf{H}$ represents the magnetic field. The electrical resistivity is assumed to be equal to some power of the current density. The motivation for our investigation

Received September 1, 1998.

2000 Mathematics Subject Classification. Primary 35K65.

Current address: Department of Pure and Applied Mathematics, Washington State University, Pullman, WA 99164. 
of (1.1)-(1.3) is twofold. On the one hand, the system (1.1) is a natural generalization of the scalar $p$-Laplacian which has been studied by many authors (see DiBenedetto [6] and the references therein). More recently; the limit solution of the $p$-Laplacian equation as $p \rightarrow \infty$ is used to describe the fast-slow diffusion model and the sandpile collapsing phenomenon (see Arronson, Evans, and Wu [1]; Evans and Gangbo [9]; Evans, Feldman, and Gariepy [8] and the references therein). On the other hand, it will be seen that the limit solution to the system (1.1)-(1.3) solves the problem of Bean's criticalstate model for a type-II superconductor (see Bean [3]). In Bean's model, the current density is assumed to be always less than or equal to a critical current, denoted by $J_{c}$. The superconductive region is characterized by the current density $J=|\nabla \times \mathbf{H}|$. When the current density is strictly less than the critical number $J_{c}$, then there is no resistance for the movement of electrons (the superconductivity is achieved) while the normal conductor region is the one where $|J|=J_{c}$. When a magnetic field is under the influence of an external force, a normal conductor material will become a superconductor one or vice versa. The motion of the interface is driven by the external force. Recently, Prigozhin in [14] reformulated the problem to a variational inequality and established the existence of a unique weak solution to the variational inequality if the space dimension is equal to two. Some numerical issues are discussed in [2] (also see a more recent paper [15]). Surprisingly, we show that the weak solution defined in [14] can be obtained as the limit of the solution to the system (1.1) as $p \rightarrow+\infty$. Here we assume that Bean's critical number $J_{c}=1$ without loss of generality. Our argument is entirely different from that of [14]. From the physical point of view, for large $p$ the electric resistivity is small in the region $S_{\varepsilon}=\{(x, t):|\nabla \times \mathbf{H}| \leq 1-\varepsilon\}$ while it is very large in $\{(x, t):|\nabla \times \mathbf{H}| \geq 1+\varepsilon\}$. Thus, the resistivity in $S_{\xi}$ becomes smaller and smaller as $p$ increases, and $S_{\varepsilon}$ becomes the superconductor region as $\varepsilon \rightarrow 0$ (no resistivity). The region $\{(x, t): 1-\varepsilon<|\nabla \times \mathbf{H}|<1+\varepsilon\}$ plays as the intermediate zone and the limit of the zone as $\varepsilon \rightarrow 0$ becomes the interface between the normal and superconductor regions.

Unlike the scalar $p$-Laplacian equation there is not much work being done about the degenerate system (1.1) as well as its limit problem. Recently, the regularity of weak solutions to a linear system analogy to (1.1) was studied in [17]. In this paper we shall first prove the existence of a weak solution to the system (1.1)-(1.3). Then we study the limit solution as $p \rightarrow \infty$ and prove that the limit solution solves a variational inequality ([14]). Many techniques are adopted from the scalar $p$-Laplacian case ([8]). To study the regularity and understand the profile of the limit solution, we consider the magnetic field $\mathbf{H}$ to be plane waves. Then it is rather surprising to see that the current density satisfies a porous medium equation. The limit problem for (1.1)-(1.3) becomes the mesa problem (see Caffarelli and Friedman [5]) for the density equation. This allows us to analyze the structure of the solution to the limit problem.

The paper is organized as follows. In $\S 2$, by using a standard variational method we show that there exists a unique weak solution to the system (1.1)-(1.3). Then we prove that the current density satisfies a porous-medium type equation when the magnetic field is assumed to be plane waves. For this special case, further regularity of the weak solution is obtained. In $\S 3$, we prove that the solution of (1.1)-(1.3) has a unique limit as $p$ goes to infinity. Moreover, the limit solution solves a variational inequality: In $\S 4$, 
we study the mesa problem with an inhomogeneous term. Similar results to the paper [5] are obtained. In $\S 5$, we use the results obtained in $\S 4$ to study the structure of the limit solution of (1.1)-(1.3).

2. Existence, uniqueness and regularity for fixed $p>2$. Let $p>2$. Define

$$
B^{d}\left(R^{3}\right)=\left\{\mathbf{G}(x) \in W^{1, p}\left(R^{3}\right): \nabla \cdot \mathbf{G}=0 \text {, a.e. } x \in R^{3}\right\} .
$$

We shall assume the following conditions on $\mathbf{H}_{0}$ and $\mathbf{F}$.

$$
\text { Assume that } \mathbf{H}_{(0)} \in B^{d}\left(R^{3}\right), \quad \mathbf{F} \in L^{2}\left(0, T ; B^{d}\left(R^{3}\right)\right) \text {. }
$$

Definition 2.1. A vector field $\mathbf{H} \in L^{2}\left(0, T ; B^{d}\left(R^{3}\right)\right)$ is said to be a weak solution of the problem (1.1)-(1.3), if the following integral identity holds:

$$
\begin{gathered}
\int_{0}^{T} \int_{R^{3}}\left[-\mathbf{H} \cdot \boldsymbol{\Phi}_{t}+|\nabla \times \mathbf{H}|^{p-2}(\nabla \times \mathbf{H}) \cdot(\nabla \times \mathbf{\Phi})\right] d x d t \\
\quad=\int_{R^{3}}\left[\mathbf{H}_{0}(x) \cdot \boldsymbol{\Phi}(x, 0)\right] d x
\end{gathered}
$$

for all $\boldsymbol{\Phi}(x, t) \in H^{1}\left(0, T ; B^{d}\left(R^{3}\right)\right)$ with $\boldsymbol{\Phi}(x, T)=0$ on $R^{3}$.

First of all, we derive some elementary energy estimates.

Lemma 2.1. Under the assumption $\mathrm{H}(2.1)$ there exist constants $C_{1}$ and $C_{2}$ such that

$$
\begin{gathered}
\sup _{[0 . T]} \int_{R^{3}}|\mathbf{H}|^{2} d x+\iint_{Q_{T}}|\nabla \times \mathbf{H}|^{p} d x d t \leq C_{1}, \\
\iint_{Q_{T}}\left|\mathbf{H}_{t}\right|^{2} d x d t+\frac{1}{p} \sup _{[0 . T]} \int_{R^{3}}|\nabla \times \mathbf{H}|^{p} d x \leq C_{2},
\end{gathered}
$$

where $C_{1}$ and $C_{2}$ depend only on known data and the upper bound of $T$.

Proof. Note that for any vector fields $\mathbf{A}, \mathbf{B} \in B^{d}$ the following identity holds:

$$
\int_{R^{3}} \mathbf{A} \cdot(\nabla \times \mathbf{B}) d x=\int_{R^{3}} \mathbf{B} \cdot(\nabla \times \mathbf{A}) d x .
$$

Taking an inner product to the system (1.1) by $\mathbf{H}$ and then using the above identity, we obtain

$$
\begin{array}{r}
\sup _{\left[0 . T^{\prime}\right]} \int_{R^{3}}|\mathbf{H}|^{2} d x+\int_{0}^{T} \int_{R^{3}}|\nabla \times \mathbf{H}|^{p} d x d t \\
\quad \leq \int_{R^{3}}\left|\mathbf{H}_{0}\right|^{2} d x+\int_{0}^{T} \int_{R^{3}} \mathbf{F} \cdot \mathbf{H} d x d t .
\end{array}
$$

By first using Cauchy's inequality and then using Gronwall's inequality, we have

$$
\begin{aligned}
& \sup _{[0 . T]} \int_{R^{3}}|\mathbf{H}|^{2} d x+\int_{0}^{T} \int_{R^{3}}|\nabla \times \mathbf{H}|^{p} d x d t \\
& \quad \leq \int_{R^{3}}\left|\mathbf{H}_{0}\right|^{2} d x+C \int_{0}^{T} \int_{R^{3}}|\mathbf{F}|^{2} d x d t .
\end{aligned}
$$

To obtain the second estimate, we take the inner product by $\mathbf{H}_{t}$ to (1.1). Similar to the first estimate one can easily derive the second estimate. 
THEOREM 2.2. Under the assumption $\mathrm{H}(2.1)$ the problem (1.1)-(1.3) has a unique weak solution. Moreover,

$$
\mathbf{H}_{t} \in L^{2}\left(Q_{T}\right), \quad \mathbf{H} \in L^{\infty}\left(0, T ; B^{d}\left(R^{3}\right)\right) .
$$

Proof. The proof is based on the standard variational method. For every $\mathbf{V} \in$ $L^{2}\left(0, T ; R^{3}\right)$, define

$$
I_{p}[\mathbf{V}]= \begin{cases}\frac{1}{p} \int_{R^{3}}|\nabla \times \mathbf{V}|^{p} d x, & \text { if } \mathbf{V} \in B^{d} \\ +\infty, & \text { otherwise }\end{cases}
$$

for a.e. $t \in(0, T]$. the system (1.1) can be reformulated by the following variational inequality:

$$
\begin{gathered}
\mathbf{F}-\mathbf{H}_{t} \in \partial I_{p}[\mathbf{H}], \quad x \in R^{3}, \quad \text { for a.e. } t \in[0, T], \\
\mathbf{H}(x, 0)=\mathbf{H}_{0}, \quad x \in R^{3},
\end{gathered}
$$

where $\partial I_{p}[\mathbf{H}]$ is the subdifferential of $I_{p}[\mathbf{V}]$ at $\mathbf{V}$ (see [16]).

It is clear that the operator $I_{p}[\mathbf{V}]$ is coercive and strictly monotonic increasing. Moreover, the operator $I_{p}$ maps from $L^{2}\left(0, T ; W^{1, p}\left(R^{3}\right)\right)$ to $L^{2}\left(0, T ; W^{-1, p^{\prime}}\left(R^{3}\right)\right)$, where $p^{\prime}=\frac{p}{p-1}$. It follows $([16])$ that the evolution problem has a unique weak solution $\mathbf{H} \in L^{2}\left(0, T ; B^{d}\right)$. Moreover, by Lemma 2.1 , the weak solution $\mathbf{H}_{t} \in L^{2}\left(Q_{T}\right)$ and $\nabla \times \mathbf{H} \in L^{p}\left(R^{3}\right)$ for a.e. $t \in[0, T]$.

Further regularity of the weak solution seems challenging since there are no local estimates such as those in [6]. However, we can obtain more regularity when the weak solution $\mathbf{H}$ is assumed to be plane waves.

From now on we shall assume that $\mathbf{H}$ depends only on $x=\left(x_{1}, x_{2}\right)$ and the component in the $z$-direction is zero, i.e., $\mathbf{H}(x, t)=\left\{h_{1}(x, t), h_{2}(x, t), 0\right\}$. In this case $Q_{T}=R^{2} \times$ $(0, T]$.

To obtain more regularity of the weak solution, we need more regularity for the known data.

Assume that $\mathbf{H}_{0} \in C^{2+\alpha}\left(R^{2}\right)$ and $\mathbf{F} \in C^{2+\alpha}\left(Q_{T}\right)$ for some $\alpha \in(0,1)$.

THEOREM 2.3. Under the assumptions $\mathrm{H}(2.1)-\mathrm{H}(2.2)$, the weak solution of the problem (1.1)-(1.3) belongs to $L^{\propto}\left(Q_{T}\right) \cap C^{1+\alpha, \beta}\left(Q_{T}\right)$ for some $\alpha, \beta \in(0,1)$. Moreover, the solution has compact support for each $t \in[0, T]$, if $\mathbf{F}$ and $\mathbf{H}_{0}$ have compact support.

Proof. Recall that $\mathbf{H}=\left\{h_{1}(x, t), h_{2}(x, t), 0\right\}$. Then it is clear that

$$
\nabla \times \mathbf{H}=\left(h_{2 x_{1}}-h_{1 x_{2}}\right) \mathbf{k}
$$

where $\mathbf{k}$ is the unit vector in the $z$-direction. It follows that

$$
\begin{aligned}
& \nabla \times\left(|\nabla \times \mathbf{H}|^{p-2} \nabla \times \mathbf{H}\right) \\
& \quad=\left\{\left[\left|h_{2 x_{1}}-h_{1 x_{2}}\right|^{p-2}\left(h_{2 x_{1}}-h_{1 x_{2}}\right)\right]_{x_{2}},-\left[\left|h_{2 x_{1}}-h_{1 x_{2}}\right|^{p-2}\left(h_{2 x_{1}}-h_{1 x_{2}}\right)\right]_{x_{1}}, 0\right\} .
\end{aligned}
$$


The system (1.1) is equivalent to the following system:

$$
\begin{aligned}
& h_{1 t}+\frac{\partial}{\partial x_{2}}\left[\left|h_{2 x_{1}}-h_{1 x_{2}}\right|^{p-2}\left(h_{2 x_{1}}-h_{1 x_{2}}\right)\right]=f_{1}\left(x_{1}, x_{2}, t\right), \\
& h_{2 t}-\frac{\partial}{\partial x_{1}}\left[\left|h_{2 x_{1}}-h_{1 x_{2}}\right|^{p-2}\left(h_{2 x_{1}}-h_{1 x_{2}}\right)\right]=f_{2}\left(x_{1}, x_{2}, t\right) .
\end{aligned}
$$

From Theorem 2.2, we know that $h_{1}\left(-\infty, x_{2}, t\right)=0$ for all $x_{2} \in R^{1}$ and $t \geq 0$. Then from Eq. (1.2), we see that

$$
h_{1}\left(x_{1}, x_{2}, t\right)=-\int_{-\infty}^{x_{1}} h_{2 x_{2}}\left(\xi, x_{2}\right) d \xi=-U_{2 x_{2}}\left(x_{1}, x_{2}, t\right),
$$

where

$$
U_{2}\left(x_{1}, x_{2}, t\right)=\int_{-\infty}^{x_{1}} h_{2}\left(\xi, x_{2}, t\right) d \xi
$$

It follows that

$$
h_{1 x_{2}}=-U_{2 x_{2} x_{2}} \quad \text { and } \quad h_{2 x_{1}}=U_{2 x_{1} x_{1}}, \quad\left(x_{1}, x_{2}, t\right) \in Q_{T} .
$$

Consequently, Eq. (2.7) becomes

$$
\frac{\partial}{\partial x_{2}}\left[-U_{2 t}+\left|\Delta U_{2}\right|^{p-2} \Delta U_{2}\right]=f_{1}\left(x_{1}, x_{2}, t\right) .
$$

Integrating over $\left(-\infty, x_{2}\right)$ yields

$$
U_{2 t}-\left|\Delta U_{2}\right|^{p-2} \Delta U_{2}=f_{2}^{*}(x, t)
$$

where

$$
f_{2}^{*}(x, t)=-\int_{-\infty}^{x_{2}} f_{1}\left(x_{1}, \xi, t\right) d \xi .
$$

Similarly, we can eliminate $h_{2}(x, t)$ to derive the following equation from (2.8):

$$
U_{1 t}-\left|\Delta U_{1}\right|^{p-2} \Delta U_{1}=f_{1}^{*}(x, y, t),
$$

where

$$
\begin{aligned}
& U_{1}(x, t)=\int_{-\infty}^{x_{1}} h_{1}\left(x_{1}, \xi, t\right) d \xi, \\
& f_{1}^{*}(x, t)=-\int_{-\infty}^{x_{1}} f_{2}\left(\xi, x_{2}, t\right) d \xi .
\end{aligned}
$$

Define

$$
u_{i}(x, t)=\Delta U_{i}(x, t), \quad g_{i}(x, t)=\Delta f_{i}^{*}(x, t), \quad i=1,2 .
$$

Then $u_{1}$ and $u_{2}$ will satisfy the following system:

$$
\begin{aligned}
u_{i t}-\Delta \psi\left(u_{i}\right) & =g_{i}(x, t), \quad(x, t) \in Q_{T}, \\
u_{i}(x, y, 0) & =u_{0 i}(x), \quad x \in R^{2}, \quad i=1,2,
\end{aligned}
$$


where

$$
\begin{array}{cc}
u_{01}(x)=\Delta \int_{-\infty}^{x_{2}} h_{01}\left(x_{1}, \xi\right) d \xi, & u_{02}(x)=\Delta \int_{-\infty}^{x_{1}} h_{02}\left(\xi, x_{2}\right) d \xi, \\
g_{1}(x, t)=-\Delta \int_{-\infty}^{x_{1}} f_{2}\left(\xi, x_{2}, t\right) d \xi, & g_{2}(x, t)=-\Delta \int_{-\infty}^{x_{2}} f_{1}\left(x_{1}, \xi, t\right) d \xi,
\end{array}
$$

while the function $\psi$ is defined by

$$
\psi(s)=|s|^{p-2} s
$$

By a result of [7] (also see [10]), there exists a unique solution $u_{i}(x, t) \in L^{2}\left(Q_{T}\right) \cap$ $L^{\infty}\left(Q_{T}\right)$. Moreover, the solution is Hölder continuous in $Q_{T}$. Furthermore, the $\operatorname{supp} u_{i}$ is compact for each $t \in[0, T]$, if $g_{i}$ and $u_{0 i}$ have compact support, $i=1,2$.

Now for each fixed $t \in(0, T]$, we solve the following elliptic problem:

$$
\begin{gathered}
\Delta U_{i}=u_{i}, \quad x \in R^{2}, \\
U_{i}(x, t)=0, \quad|x| \rightarrow+\infty .
\end{gathered}
$$

By applying the standard elliptic theory [11], we know that the problem (2.13)-(2.14) admits a unique solution $U_{i}(x, t) \in C^{2+a .0}\left(R^{2}\right)$. Now we claim that $U_{i}(x, t)$ is also Hölder continuous with respect to $t$. Indeed, for small $\Delta t$ we define

$$
U_{i}^{*}=\frac{U_{i}\left(x, t+\Delta t-U_{i}(x, t)\right.}{|\Delta t|^{\alpha / 2}} .
$$

Then $U_{i}^{*}$ solves the following problem:

$$
\begin{gathered}
\Delta U_{i}^{*}=\frac{u_{i}(x, t+\Delta t)-u_{i}(x, t)}{|\Delta t|^{\alpha / 2}}, \quad x \in R^{2}, \\
U_{i}^{*}(x, t)=0, \quad|x| \rightarrow+\infty .
\end{gathered}
$$

Since $u_{i}(x, t)$ is Hölder continuous with respect to $t$, we apply $L^{p}$-theory for elliptic equations to obtain

$$
\left\|U_{i}^{*}\right\|_{W_{p}^{2}\left(B_{R}(0)\right)} \leq C
$$

where $B_{R}(0)=\left\{x \in R^{2}:|x|<R\right\}$ and the constant $C$ depends only on $p$ and the Hölder norm of $u_{i}$ and $R$, but is independent of $\Delta t$.

By Sobolev's embedding, we know that for every $R>0$ there exist constants $C(R)$ and $\alpha \in(0,1)$ such that

$$
\left\|U_{i}^{*}\right\|_{C^{1+a}\left(B_{R}(0)\right)} \leq C(R)
$$

A compactness argument yields that $U_{i}(x, t)$ and $U_{i x_{j}}$ are Hölder continuous with respect to $t$ for each $i$ and $j$, where $i=1,2$ and $j=1,2$. It follows that $h_{1}(x, t)=-\frac{\partial}{\partial x_{2}} U_{2}(x, t)$ and $h_{2}(x, t)=\frac{\partial}{\partial x_{1}} U_{1}(x, t)$ are Hölder continuous with respect to $t$. Finally, an interpolation lemma ([13]) implies that $h_{i}$ is Hölder continuous with respect to $t$ with Hölder exponent $\beta$ for some $\beta \in(0,1)$. 
3. The limit solution as $p \rightarrow \infty$. In this section we shall show that the solution of (1.1)-(1.3) has a limit as $p \rightarrow \infty$, which solves a variational inequality if the initial current density is less than the Bean critical value.

$$
\text { Assume that the initial field } \mathbf{H}_{0} \text { satisfies }\left\|\nabla \times \mathbf{H}_{0}\right\|_{L^{\infty}\left(R^{3}\right)} \leq 1 \text {. }
$$

LEMMA 3.1. Under the assumptions $\mathrm{H}(2.1)$ and $\mathrm{H}(3.1)$, the energy estimates (2.2)-(2.3) hold, where the constants $C_{1}$ and $C_{2}$ are independent of $p$.

The proof is the same as for Lemma 2.1 except that the assumption $\mathrm{H}(3.1)$ will ensure that $C_{1}$ and $C_{2}$ are independent of $p$.

We shall denote by $\mathbf{H}^{(p)}$ the solution of the system (1.1)-(1.3). From Lemma 3.1, we know that every subsequence $\mathbf{H}^{\left(p^{\prime}\right)}$ with $p^{\prime} \rightarrow+\infty$ has a subsequence $\mathbf{H}^{\left(p^{\prime \prime}\right)}$ that converges to a limit, denoted by $\mathbf{H}^{(\infty)}$, weakly in $*-L^{\infty}(\Omega \times(0, T])$ for any bounded domain $\Omega \subset R^{3}$. Moreover,

$$
\begin{aligned}
\nabla \times \mathbf{H}^{\left(p^{\prime \prime}\right)} & \rightarrow \nabla \times \mathbf{H}^{(\infty)}, \\
\mathbf{H}_{t}^{\left(p^{\prime \prime}\right)} & \rightarrow \mathbf{H}_{t}^{(\infty)}
\end{aligned}
$$

weakly in $L^{2}(\Omega \times(0, T))$. Furthermore, since $\nabla \cdot \mathbf{H}^{(p)}=0, \mathbf{H}^{(p)} \in L^{2}\left(0, T ; B^{d}\right)$, and $\mathbf{H}_{t}^{(p)} \in L^{2}\left(Q_{T}\right)$, it follows that

$$
\mathbf{H}^{\left(p^{\prime \prime}\right)} \rightarrow \mathbf{H}^{(\infty)} \quad \text { a.e. in } Q_{T} .
$$

LEMmA 3.2. The limit $\mathbf{H}^{(x)}$ satisfies the following estimate:

$$
\underset{Q_{T}}{\operatorname{ess} \sup }\left|\nabla \times \mathbf{H}^{(x)}\right| \leq 1 .
$$

Proof. From the proof of Lemma 2.1, we see that

$$
\int_{0}^{T} \int_{R^{3}}|\nabla \times \mathbf{H}|^{p} p d x d t \leq C p
$$

where $C$ is a constant that is independent of $p$.

Similar to [8], for every small $\delta>0$ we define

$$
A_{\delta}=\left\{(x, t) \in Q_{T}:\left|\nabla \times \mathbf{H}^{(p)}\right| \geq 1+\delta\right\} .
$$

Then,

$$
\begin{aligned}
(1+\delta)\left|A_{\delta}\right| & \leq \iint_{A_{\delta}}\left|\nabla \times \mathbf{H}^{(p)}\right| d x d t \\
& \leq \liminf _{p_{i} \rightarrow \infty} \iint_{A_{\delta}}\left|\nabla \times \mathbf{H}^{\left(p_{i}\right)}\right| d x d t \\
& \leq \liminf _{p_{i} \rightarrow \infty}\left(\iint_{A_{\delta}}\left|\nabla \times \mathbf{H}^{\left(p_{i}\right)}\right|^{p_{i}} d x d t\right)^{\frac{1}{p_{i}}}\left|A_{\delta}\right|^{1-\frac{1}{p_{i}}} \\
& \leq\left|A_{\delta}\right| .
\end{aligned}
$$

It follows that

$$
\left|A_{\delta}\right|=0 .
$$

The desired result follows since $\delta$ is arbitrary. 
Before stating the main result in this section, we define another functional. Let

$$
K=\left\{\mathbf{V} \in L^{2}\left(Q_{T}\right) ; \nabla \cdot \mathbf{V}=0\right\} .
$$

For every $\mathbf{V} \in K$, define $I_{p}[\mathbf{V}]$ as in Sec. 2 for a.e. $t \in(0, T]$. Similarly, we define

$$
I_{\infty}[\mathbf{V}]= \begin{cases}0, & \text { if } \mathbf{V} \in K, \quad|\nabla \times \mathbf{V}| \leq 1, \\ +\infty, & \text { otherwise. }\end{cases}
$$

Theorem 3.3. Under the assumptions $\mathrm{H}(2.1)$ and $\mathrm{H}(3.1)$ the limit function $\mathbf{H}^{(\infty)}$ is unique and satisfies the following variational problem:

$$
\begin{gathered}
\mathbf{F}-\mathbf{H}_{t} \in \partial I_{\infty}[\mathbf{H}], \quad \text { for a.e. } t>0, \\
\mathbf{H}(x, 0)=\mathbf{H}_{0}(x), \quad x \in R^{3},
\end{gathered}
$$

that is, for a.e. $t \in[0, T]$,

$$
\begin{aligned}
I_{\infty}[\mathbf{V}] & -I_{\infty}\left[\mathbf{H}^{(\infty)}\right] \\
\geq & \int_{R^{3}}\left(\mathbf{F}-\mathbf{H}_{t}^{(\infty)}\right) \cdot\left(\mathbf{V}-\mathbf{H}^{(\infty)}\right) d x \quad \text { a.e. } t \geq 0,
\end{aligned}
$$

for all $\mathbf{V} \in K$ with $\mathbf{V}_{t} \in L^{2}\left(Q_{T}\right)$.

Proof. Since the limit is unique we may assume that the whole sequence $\mathbf{H}^{(p)}$ converges to $\mathbf{H}^{(\infty)}$. For simplicity, we denote by $\mathbf{H}$ the limit solution. Note that the system (1.1) is equivalent to the following variational form:

$$
\mathbf{F}-\mathbf{H}_{t} \in \partial I_{p}[\mathbf{H}] \text {. }
$$

By the definition of $I_{\infty}[\mathbf{V}]$, we only need to verify the inequality for all $\mathbf{V} \in L^{2}\left(Q ; R^{2}\right)$ with $|\nabla \times \mathbf{V}| \leq 1$ a.e. $t \in[0, T]$. On the other hand, Lemma 3.2 implies that $I_{\infty}[\mathbf{H}]=0$ for a.e. $t \in[0, T]$. Thus, the inequality (3.3) is equivalent to

$$
\int_{R^{3}}\left(\mathbf{F}-\mathbf{H}_{t}\right) \cdot(\mathbf{V}-\mathbf{H}) d x \leq 0 .
$$

Since $\mathbf{H}^{(p)}$ solves the variational problem (2.4)-(2.5), we see that

$$
\begin{aligned}
I_{p}[\mathbf{V}] & \geq I_{p}\left[\mathbf{H}^{(p)}\right]+\int_{R^{2}}\left(\mathbf{F}-\mathbf{H}_{t}^{(p)}\right) \cdot\left(\mathbf{V}-\mathbf{H}^{(p)}\right) d x \\
& \geq \int_{R^{3}}\left(\mathbf{F}-\mathbf{H}_{t}^{(p)}\right) \cdot\left(\mathbf{V}-\mathbf{H}^{(p)}\right) d x .
\end{aligned}
$$

By assumptions on $\mathbf{V}$, we have

$$
\lim _{p \rightarrow \infty} I_{p}[\mathbf{V}]=0
$$

On the other hand, since $\mathbf{H}^{(p)} \rightarrow \mathbf{H}$ a.e. and $\mathbf{H} \in L^{2}\left(Q_{T}\right)$, it follows that

$$
\int_{R^{3}}\left(\mathbf{F}-\mathbf{H}_{t}^{(p)}\right) \cdot\left(\mathbf{V}-\mathbf{H}^{(p)}\right) d x \rightarrow \int_{R^{3}}\left(\mathbf{F}-\mathbf{H}_{t}\right) \cdot(\mathbf{V}-\mathbf{H}) d x
$$

After taking the limit in the inequality (3.5), we obtain the desired inequality (3.3). To verify the initial condition, we note that

$$
\int_{R^{3}}\left|\mathbf{H}(x, t)-\mathbf{H}_{0}(x)\right|^{2} d x \leq C \sqrt{t} \rightarrow 0
$$


as $t \rightarrow 0$, since $\left\|\mathbf{H}_{t}\right\|_{L^{2}\left(Q_{T}\right)} \leq C$.

Now we prove the uniqueness. Suppose there are two solutions $\mathbf{H}$ and $\mathbf{H}^{*}$ to the limit problem. Then by Lemma 3.2 we know that

$$
|\nabla \times \mathbf{H}| \leq 1, \quad\left|\nabla \times \mathbf{H}^{*}\right| \leq 1 .
$$

We choose $\mathbf{H}$ and $\mathbf{H}^{*}$ as test functions in (3.3), respectively, to obtain

$$
\begin{gathered}
\int_{R^{3}}\left(\mathbf{F}-\mathbf{H}_{t}\right) \cdot(\mathbf{V}-\mathbf{H}) d x \leq 0, \\
\int_{R^{3}}\left(\mathbf{F}-\mathbf{H}_{t}^{*}\right) \cdot\left(\mathbf{V}-\mathbf{H}^{*}\right) d x \leq 0 .
\end{gathered}
$$

Consequently, one obtains

$$
\int_{R^{3}}\left(\mathbf{H}^{*}-\mathbf{H}\right)_{t} \cdot\left(\mathbf{H}-\mathbf{H}^{*}\right) d x \leq 0 \quad \text { a.e. } t \geq 0
$$

It follows that

$$
\frac{d}{d t}\left\|\mathbf{H}-\mathbf{H}^{*}\right\|_{L^{2}\left(Q_{T}\right)} \leq 0,
$$

which implies the uniqueness.

REMARK 3.1. The solution obtained in [14] is essentially the same as the limit solution in Theorem 3.3 if the space dimension is equal to 2 .

Now we investigate further regularity of the limit solution when $\mathbf{H}$ is a plane wave.

TheOrem 3.4. If the space dimension is equal to 2 , then the limit solution $\mathbf{H}^{(\infty)}$ is globally bounded and Hölder continuous in $Q_{T}$.

Proof. When $\mathbf{H}^{(p)}=\left\{h_{1}, h_{2}, 0\right\}$, we can study the regularity for $u_{i}^{(\infty)}$. Recall that for each $t \in[0, T]$,

$$
\begin{gathered}
\Delta U_{i}^{(p)}=u_{i}^{(p)}(x, t), \quad x \in R^{2}, \\
U_{i}^{(p)}(x, t)=0, \quad|x| \rightarrow \infty .
\end{gathered}
$$

Since $\left\|u_{i}^{(p)}\right\|_{L^{\infty}\left(Q_{T}\right)} \leq 1$, the elliptic theory implies that for any $q>1$,

$$
\left\|U_{i}^{(p)}\right\|_{W^{2, q}\left(B_{R}(0)\right)} \leq C,
$$

where $C$ depends only on $R$ and $q$, but not on $p$. It follows by Sobolev's embedding and a compactness argument that for any $\alpha \in(0,1), U_{i}^{(\infty)}(x, t) \in C^{1+\beta, 0}\left(Q_{T}\right)$ for any $\beta \in(0,1)$ and each $t \in[0, T]$. It follows from the definition that $\mathbf{H}^{(\infty)} \in C^{\alpha, 0}\left(Q_{T}\right)$.

Next we derive the regularity of $U_{i}^{(\infty)}(x, t)$ with respect to $t$. Recall that $U_{i}^{(p)}$ satisfies Eq. (3.6). Note that

$$
\left\|u_{i t}\right\|_{L^{1}\left(Q_{T}\right)} \leq C
$$

where $C$ is independent of $p$. It follows that for any $q \in(1,2)$,

$$
\left\|U_{i t}^{(p)}\right\|_{W^{1, q}\left(Q_{T}\right)} \leq C,
$$

where $C$ is independent of $p$. Since $\mathbf{H}^{(\infty)} \in C^{1+\beta, 0}\left(Q_{T}\right)$, it follows by the interpolation result ([13]) that the limit function $U_{i}^{(\infty)}$ and $U_{i x_{l}}^{(\infty)}$ are Hölder continuous with respect to $t$. 
4. The mesa problem with an inhomogeneous term. As we have seen in Sec. 2 , the limit problem for the system (1.1)-(1.3) becomes the mesa problem for the current density if the space dimension is equal to 2 . Throughout this section we shall study the mesa problem with an inhomogeneous term. By employing similar techniques to [5], we derive the profile of the limit solution to the mesa problem. The result will be used to understand the initial collapsing and afterwards evolution process for the current density of the limit solution $\mathbf{H}^{(\infty)}$ in the case of two space dimensions (see Sec. 5 below).

Consider the mesa problem with an inhomogeneous term:

$$
\begin{gathered}
u_{t}-\Delta u^{m}=g(x, t), \quad(x, t) \in R^{n} \times(0, \infty), \\
u(x, 0)=f(x), \quad x \in R^{n} .
\end{gathered}
$$

Assume that $f(x) \in L^{1}\left(R^{n}\right) \cap L^{\infty}\left(R^{n}\right)$ and

$$
g(x, t) \in L^{1}\left(R^{n} \times(0, \infty)\right) \cap L^{\infty}\left(R^{n} \times(0, \infty)\right) .
$$

Moreover, $f(x) \geq 0$ and $g(x, t) \geq 0$ on $Q_{T}=R^{n} \times(0, T]$.

LEMMA 4.1. Let

$$
M=\|f\|_{L^{x}\left(R^{n}\right)}+T\|g\|_{L^{x}\left(Q_{T}\right)} .
$$

Then

$$
\left\|u^{(m)}\right\|_{L \times\left(Q_{T}\right)} \leq M .
$$

Moreover, if $M<1$, then $u^{(m)}$ has a unique limit $u^{(x)}(x, t)$ as $m \rightarrow \infty$ and

$$
u^{(x)}(x, t)=f(x)+\int_{0}^{t} g(x, \tau) d \tau, \quad(x, t) \in Q_{T} .
$$

Proof. As a first step, we may assume that $f(x)$ and $g(x, t)$ have compact support. Then $u^{(m)}(x, t)$ has compact support for each $t \in[0, T]$ and any $m>1$. Let $R$ be sufficiently large such that $u^{(m)}(x, t)=0$ on $\partial B_{R}(0) \times[0, T]$. We may also assume that $u^{(m)}$ is smooth. Define the operator $L$ as follows:

$$
L[u]=m u^{m-1} \Delta u+m(m-1) u^{m-2}|\nabla u|^{2}-u .
$$

Let

$$
v(x, t)=\|f\|_{L^{\times}\left(R^{n}\right)}+t\|g\|_{L^{\times}\left(Q_{T}\right)} .
$$

Then

$$
L\left[u^{(m)}-v\right]=g(x, t)+\|g\|_{L^{x}\left(Q_{T}\right)} \geq 0 .
$$

On the parabolic boundary of $B_{R}(0) \times[0, T]$,

$$
u^{(m)}-v \leq 0 .
$$

By the comparison principle, we can see that

$$
u^{(m)}(x, t) \leq v(x, t) \leq M .
$$

The lower bound of $u^{(m)}$ can be derived similarly. In general, we use the standard approximation (see p. 715 of [5], for example) to derive the desired estimate. 
To prove the second conclusion, we note that

$$
\int_{R^{n}} u^{(m)}(x, t) d x-\int_{R^{n}} f(x) d x=\int_{R^{n}} \int_{0}^{t} g(x, \tau) d \tau d x
$$

It follows that

$$
\left\|u^{(m)}\right\|_{L^{1}\left(R^{n}\right)} \leq\|f\|_{L^{1}\left(R^{n}\right)}+\|g\|_{L^{1}\left(Q_{T}\right)} .
$$

For any fixed smooth function $\phi(x)$, we have

$$
\begin{gathered}
\int_{R^{n}} \Delta\left(u^{(m)}\right)^{m} \phi(x) d x=\int_{R^{n}}\left(u^{(m)}\right)^{m} \Delta \phi(x) d x \\
\leq C M^{m-1}\left\|u^{(m)}\right\|_{L^{1}\left(R^{n}\right)} \rightarrow 0 \text { as } m \rightarrow \infty .
\end{gathered}
$$

It follows that

$$
\int_{R^{n}} u^{(x)}(x, t) \phi(x) d x=\int_{R^{n}} f(x) \phi(x) d x+\int_{R^{n}}\left[\int_{0}^{t} g(x, \tau) d \tau\right] \phi(x) d x .
$$

Thus,

$$
u^{(x)}(x, t)=f(x)+\int_{0}^{t} g(x, \tau) d \tau
$$

for a.e. $x \in R^{n}, t \geq 0$.

From Lemma 4.1, we know that $u^{(m)}$ is uniformly bounded. Then for any fixed $t>0$ every sequence $u^{\left(m^{\prime}\right)}(x, t)$ with $m^{\prime} \rightarrow \infty$ has a subsequence $u^{\left(m^{\prime \prime}\right)}$ with $m^{\prime \prime} \rightarrow \infty$ such that

$$
u^{\left(m^{\prime \prime}\right)} \rightarrow u^{(\infty)}(x, t) \quad \text { weakly in } *-L^{\infty}(\Omega) .
$$

In general $u^{(\infty)}(x, t)$ may not be unique. However, we show that there is a unique limit for $u^{(\infty)}(x, t)$ as $t \rightarrow 0+$.

LEMMA 4.2. Under the assumption $\mathrm{H}(4.1)$, the following estimate holds:

$$
\left\|u_{t}\right\|_{L^{1}\left(R^{n}\right)} \leq \frac{1}{t}\|g\|_{L^{1}\left(R^{n}\right)}+\left[\frac{1}{t(m-1)}\|u\|_{L^{1}\left(R^{n}\right)}+\|f\|_{L^{1}\left(R^{n}\right)}\right] .
$$

Proof. Let $\hat{u}(x, t)=\lambda^{\frac{1}{m-1}} u(x, \lambda t)$. Then it is easy to see that $\hat{u}$ solves the following problem:

$$
\begin{gathered}
\hat{u}_{t}-\Delta \hat{u}^{m}=\lambda^{\frac{m}{m-1}} g(x, t), \quad(x, t) \in R^{n} \times(0, \infty), \\
\hat{u}(x, 0)=\lambda^{\frac{1}{m-1}} f(x), \quad x \in R^{n} .
\end{gathered}
$$

By $L^{1}$-stability for the porous medium equation ([4]) we know that

$$
\|u-\hat{u}\|_{L^{1}\left(R^{n}\right)} \leq C\left[\left|\lambda^{\frac{m}{m-1}}-1\right|\|g\|_{L^{1}\left(R^{n}\right)}+\left|\lambda^{\frac{1}{m-1}-1}\right|\|f\|_{L^{1}\left(R^{n}\right)}\right]
$$

where $C$ depends only on $n$, but not on $m$. By taking the limit as $\lambda \rightarrow 1+$, we obtain

$$
\begin{aligned}
& \left\|\frac{1}{m-1} u+t u_{t}\right\|_{L^{1}\left(R^{n}\right)} \\
& \quad \leq \frac{m}{m-1}\|g\|_{L^{1}\left(R^{n}\right)}+\frac{C}{m-1}\|f\|_{L^{1}\left(R^{n}\right)},
\end{aligned}
$$

which yields the desired estimate. 
LEMma 4.3. The limit solution satisfies

$$
0 \leq u^{(\infty)} \leq 1 \quad \text { in } Q_{T} .
$$

Proof. For any $\delta>0$, we multiply Eq. (4.1) by $\hat{u}=[u-(1+\delta)]^{+}$to obtain that

$$
\begin{aligned}
& \int_{R^{n}}|\hat{u}|^{2} d x+\int_{0}^{T} \int_{R^{n}} m u^{m-1}|\nabla \hat{u}|^{2} d x d t \\
& \quad \leq \int_{R^{n}}|\hat{u}(x, 0)|^{2} d x+\int_{0}^{T} \int_{R^{n}}|g|^{2} d x d t \leq C,
\end{aligned}
$$

where $C$ is independent of $m$.

It follows that

$$
\int_{0}^{T} \int_{R^{n}}|\nabla \hat{u}|^{2} d x d t \leq \frac{C}{m}
$$

Fatou's lemma implies that

$$
\left|\left\{(x, t): u^{(\infty)} \geq 1+\delta\right\}\right|=0,
$$

which yields the desired result since $\delta$ is arbitrary.

From Lemma 4.3, we see that there must exist an initial collapsing that occurs instantly if $\|f\|_{L^{\infty}\left(R^{n}\right)}>1$. The external force should play no role in this collapsing process. The basic strategy is to separate the initial collapsing and the evolution processes.

$$
\text { Let } L=\|f\|_{L^{\infty}\left(R^{n}\right)}>1 \text {. }
$$

TheOREM 4.4. Under the assumptions $\mathrm{H}(4.1)-\mathrm{H}(4.2), u^{(m)}(x, t)$ has a unique limit as $m \rightarrow \infty$ and $t \rightarrow 0+$. Moreover,

$$
u^{(\infty)}(x, 0+)=v^{(\infty)}(x), \quad x \in R^{n},
$$

where $v^{(\infty)}(x)$ is the limit function of the solution $v^{(m)}$ to $(4.1)-(4.2)$ with $g(x, t) \equiv 0$.

Proof. Let $v^{(m)}$ be the solution of (4.1)-(4.2) with $g(x, t)=0$. Let $\phi(x)$ be any bounded function with compact support. Let $\left(m^{\prime}, t_{m^{\prime}}\right)$ and $\left(m^{\prime \prime}, t_{m^{\prime \prime}}\right)$ be any two sequence pairs that converge to $(\infty, 0+)$, respectively, as $m \rightarrow \infty$. For any bounded function $\phi$,

$$
\begin{aligned}
& \left|\int_{R^{n}} \phi\left[u^{\left(m^{\prime}\right)}\left(x, t_{m^{\prime}}\right)-v^{\left(m^{\prime \prime}\right)}\left(x, t_{m^{\prime \prime}}\right)\right] d x\right| \\
& \quad \leq\|\phi\|_{L^{\infty}\left(R^{n}\right)} \int_{R^{n}}\left[\int_{0}^{t_{m^{\prime}}}\left|u_{t}^{\left(m^{\prime}\right)}\right| d \tau+\int_{0}^{t_{m^{\prime \prime}}}\left|v_{t}^{\left(m^{\prime \prime}\right)}\right| d \tau\right] d x \\
& \quad \leq C\|\phi\|_{L^{\infty}\left(R^{n}\right)}\left[\int_{0}^{\max \left\{t_{m^{\prime}}, t_{m^{\prime \prime}}\right\}} \int_{R^{n}}|g| d x d \tau+\frac{C}{m^{\prime}-1}+\frac{C}{m^{\prime \prime}-1}\right] \\
& \quad \rightarrow 0,
\end{aligned}
$$

as $m^{\prime}, m^{\prime \prime} \rightarrow \infty$. Let the limit function be denoted by $u^{(\infty)}(x, 0+)$.

From [5], we know that $v^{(m)}(x, t)$ converges to a unique limit $v^{(\infty)}(x)$ as $m \rightarrow \infty$. Particularly, we see that

$$
v^{\left(m^{\prime \prime}\right)}\left(x, t_{m^{\prime \prime}}\right) \rightarrow v^{(\infty)}(x, 0+) \equiv v^{(\infty)}(x)
$$


as $m^{\prime \prime} \rightarrow \infty$.

It follows that

$$
\int_{R^{n}} \phi u^{(\infty)}(x, 0+) d x=\int_{R^{n}} \phi v^{(\infty)}(x, 0+) d x .
$$

Since $\phi(x)$ is arbitrary, we see that

$$
u^{(\infty)}(x, 0+)=v^{(\infty)}(x, 0+)=v^{(\infty)}(x), \quad \text { a.e. } x \in R^{n} .
$$

REMARK 4.1. Physically, Lemma 4.3 means that the collapsing process happens instantly before the force term $g(x, t)$ affects the evolution process.

To see the profile of the limit solution for $t>0$, we may start with $u^{(\infty)}(x, 0+)$ as an initial value. Therefore, without loss of generality, from now on we assume that

$$
L=\|f\|_{L^{\infty}\left(R^{n}\right)} \leq 1 .
$$

Lemma 4.5. Assume that $\|f\|_{L^{\infty}\left(R^{n}\right)} \leq 1$. Then

$$
V_{m}=\frac{m}{m-1}\left(u^{(m)}\right)^{m-1}
$$

is uniformly bounded.

Proof. Without loss of generality, we may assume that $V_{m}$ is smooth and that $\operatorname{supp} V_{m}$ $\subset B_{R}(0)$ for all $t \in[0, T]$. A direct calculation shows that $V_{m}$ satisfies

$$
\begin{aligned}
V_{m, t} & =(m-1) V_{m} \Delta V_{m}+\left|\nabla V_{m}\right|^{2}+V_{m}^{\beta} g(x, t), \quad x \in B_{R}, t \in(0, T], \\
V_{m}(x, t) & =0, \quad(x, t) \in \partial B_{R}(0) \times[0, T], \\
V_{m}(x, 0) & =\frac{m}{m-1} f(x)^{m-1}, \quad x \in R^{n},
\end{aligned}
$$

where $\beta=\frac{m-2}{m-1}<1$. If $V_{m}$ attains a positive maximum $M>1$, then the coefficient of $V_{m}, g / V_{m}^{1-\beta}$, has an upper bound that is independent of $m$. The strong maximum principle implies that $V_{m}$ cannot take a positive maximum that is greater than 1 in $B_{R}(0) \times(0, T]$. It follows that

$$
V_{m} \leq \max \left\{1, \max _{B_{R}(0)} V_{m}(x, 0)\right\} \leq \frac{m}{m-1} \leq 2 .
$$

LEMMA 4.6. $u^{(m)}$ has a uniform compact support.

Proof. By Lemma 4.5, $V_{m}$ is uniformly bounded. Now consider the special Barenblatt solution

$$
\hat{u}=t^{-\frac{n}{n(m-1)+2}}\left[\xi_{0}^{2}-\frac{m-1}{2[n(m-1)+2]}|x|^{2} t^{-\frac{2}{n(m-1)+2}}\right]_{+}^{\frac{1}{m-1}},
$$

where $(s)_{+}=\max \{s, 0\}$ and

$$
\xi_{0}=\left[\frac{M}{w_{n}}\left(\frac{(2(n(m-1)+2}{m-1}\right)^{-\frac{n}{2}}\left(\int_{0}^{1}\left(1-y^{2}\right)^{\frac{1}{m-1}} y^{n-1} d y\right)^{-1}\right]^{\frac{m-1}{n(m-1)+2}}
$$


Assume that $\operatorname{supp} f, \operatorname{supp} g \subset B_{R}(0)$ for some large $R$ and for every $t \in[0, T]$. By choosing $M$ sufficiently large and using the comparison principle in the domain $\left(R^{n} \backslash B_{R}(0)\right) \times(0, T]$, we obtain the desired result.

Assume that $f(x) \in C^{1}\left(R^{n}\right)$ is radially symmetric with compact support and $f_{r}<0$ in $R^{n} \backslash\{0\} \cap \operatorname{supp} f$. Assume that $g(x, t) \in C^{2.1}\left(R^{n} \times[0, T]\right)$ is radially symmetric in $x$ and $g_{r} \leq 0$ in $R^{n} \backslash\{0\} \cap \operatorname{supp} g$ for each $t \in[0, T]$. Moreover, $\Delta f^{m}+g(x, 0) \geq 0$.

LEmmA 4.7. Under the assumptions $\mathrm{H}(4.1)-\mathrm{H}(4.3), u_{r}^{(m)} \leq 0$ and $u_{t}^{(m)} \geq 0$.

Proof. This can be derived by a simple application of the maximum principle.

THEOREM 4.8. Under the assumptions $\mathrm{H}(4.1)$ and $\mathrm{H}(4.3)$, the limit solution $u^{(\infty)}$ is uniquely determined and

$$
u^{(x)}(x, t)=\left\{\begin{array}{l}
1, \quad \text { in }\{(x, t): w(x, t)>0\} \\
f(x)+\int_{0}^{t} g(x, \tau) d \tau, \quad \text { in }\{(x, t): w(x, t)=0\}
\end{array}\right.
$$

where $w(x, t)$ is the unique solution of the variational inequality

$$
\begin{gathered}
-\Delta w \geq f(x)+\int_{0}^{t} g(x, \tau) d \tau-1, \\
w(x, t) \geq 0, \\
w(x, t)\left[\Delta w+f(x)+\int_{0}^{t} g(x, \tau) d \tau-1\right]=0
\end{gathered}
$$

for a.e. $(x, t) \in R^{n} \times(0, T]$.

Proof. Since the proof closely follows the argument of [5] (Theorems 5.2-5.3), we only give a brief outline here. First of all we recall that $\|f\|_{L^{x}\left(R^{n}\right)} \leq 1$. Since $f_{r} \leq 0$, we may further assume that $f(x)=1$ on a small ball, say, $B_{\delta}(0)$ for some small $\delta \geq 0$. Since $g(x, t) \geq 0$, by the comparison principle (see [5], Theorem 3.2) we see that $u^{(\infty)}(x, t)=1$ for a.e. $x \in B_{\delta}$ and $t \in[0, T]$.

Step 1 . For each $t \in[0, T]$, define

$$
N_{t}=\left\{x: f(x)+\int_{0}^{t} g(x, \tau) d \tau=1\right\} .
$$

By the comparison principle, it is clear that $N_{t_{1}} \subset N_{t_{2}}$ if $t_{1}<t_{2}$. We claim that

$$
u^{(x)}(x, t)=1 \quad \text { for a.e. all } x \in N_{t} .
$$

Indeed, we replace $f$ and $g$ by $f_{\varepsilon}=\min \{1-\varepsilon, f\}$ and $g_{\varepsilon}$, respectively, for small $\varepsilon>0$. Let $u_{\varepsilon}^{(m)}$ and $u_{\varepsilon}^{(x)}$ be the solutions of (4.1)-(4.2) corresponding to $f_{\varepsilon}$ and $g_{\varepsilon}$, respectively. Then Lemma 4.1 yields

$$
u_{\varepsilon}^{(x)}(x, t)=f_{\varepsilon}(x)+\int_{0}^{t} g_{\varepsilon}(x, \tau) d \tau
$$

for a.e. all $x \in R^{n}$. On the other hand, the comparison principle implies

$$
u^{(m)}(x, t) \geq u_{\varepsilon}^{(m)}(x, t) .
$$


It follows that

$$
u^{(\infty)}(x, t) \geq f_{\varepsilon}(x)+\int_{0}^{t}(g-\varepsilon) d \tau .
$$

It follows by the definition of $N_{t}$ that

$$
u^{(\infty)}(x, t)=1, \quad \text { as } \varepsilon \rightarrow 0 .
$$

for a.e. $x \in N_{t}$.

Step 2. Let

$$
\begin{aligned}
S_{t} & =\left\{x: u^{(\infty)}(x, t)<1\right\}, \\
S_{t}^{*} & =\left\{x \in S_{t}: S_{t} \text { has Lebesgue density } 1 \text { at } x\right\} .
\end{aligned}
$$

Assume that for any $x_{0} \in S_{t}^{*}$ and $t_{0} \in(0, T], u^{(\infty)}\left(x_{0}, t_{0}\right) \leq \theta_{0}<1$. Then for any $\theta \in\left(\theta_{0}, 1\right), \delta>0$, there exists an $m_{0}$ such that

$$
\inf _{x \in Q_{\delta}\left(x_{0}, t_{0}\right)} u^{\left(m^{\prime}\right)}(x, t) \leq \theta,
$$

if $m^{\prime}>m_{0}$.

The proof is almost the same as the proof of Theorem 3.3 in [5]. We skip it here.

Step 3. If $x_{0} \in S^{*}$ with $u^{(\infty)}\left(x_{0}, t_{0}\right) \leq \theta_{0}<1$, then

$$
u^{(\infty)}(x, t)=f(x)+\int_{0}^{t} g(x, \tau) d \tau
$$

in the $\left(R^{n} \backslash B_{r_{0}}(0)\right) \times\left[0, T_{0}\right]$, where $r_{0}=\left|x_{0}\right|$.

This step can be proved by using Step 3 and Lemma 4.7. Indeed, by Step 2 we know that for any $\theta \in\left(\theta_{0}, 1\right)$ and any $\delta>0$ there exists $m_{0}$ such that

$$
\inf _{B_{\delta}\left(x_{0}\right)} u^{\left(m^{\prime}\right)}\left(x, t_{0}\right)<\theta
$$

if $m^{\prime}>m_{0}$. Suppose that the minimum of $u^{\left(m^{\prime}\right)}$ is attained at a point $\left(x^{*}, t_{0}\right)$ in $B_{\delta}\left(x_{0}\right)$. Since $u_{r}^{(m)} \leq 0$ and $u_{t}^{(m)} \geq 0, x^{*}$ must occur on the boundary of $B_{\delta}\left(x_{0}\right)$ and, if $m^{\prime}>m_{0}$, then

$$
u^{\left(m^{\prime}\right)}(x, t) \leq \theta
$$

for all $(x, t) \in\left(R^{n} \backslash B_{r_{0}+\delta}(0)\right) \times\left[0, t_{0}\right]$, where $r_{0}=\left|x_{0}\right|$. It follows by Lemma 4.1 and Lemma 4.7 that

$$
u^{(\infty)}(x, t)=f(x)+\int_{0}^{t} g(x, \tau) d \tau
$$

for all $(x, t) \in\left(R^{n} \backslash B_{r_{0}+\delta}(0)\right) \times\left[0, t_{0}\right]$. Finally, since $\delta$ is arbitrary, we conclude the desired result.

Step 4.

$$
u^{(\infty)}(x, t)=\chi_{N_{t}}+\left[f(x)+\int_{0}^{t} g(x, \tau) d \tau\right] \chi_{R^{n} \backslash N_{t}} .
$$

Indeed, by Step 1 to Step 3, there exists a function $0 \leq \hat{f}(x, t)<1$ such that

$$
u^{(\infty)}(x, t)=\chi_{N_{t}}+\hat{f}(x, t) \chi_{R^{n} \backslash N_{t}} .
$$


If at a point $\left(x_{0}, t_{0}\right)$,

$$
f\left(x_{0}\right)+\int_{0}^{t_{0}} g(x, \tau) d \tau<1
$$

then by Lemma 4.1 and Lemma 4.7

$$
u^{(\infty)}(x, t)=f(x)+\int_{0}^{t} g(x, \tau) d \tau
$$

for all $x \in R^{n} \backslash B_{r_{0}}(0)$. It follows that

$$
\hat{f}(x, t)=f(x)+\int_{0}^{t} g(x, \tau) d \tau .
$$

Step 5 .

$$
N_{t}=\{x: w(x, t)>0\},
$$

where $w(x, t)$ is the solution of the variational inequality (4.3)-(4.5).

First of all, by Lemma 4.6 we know that $u^{(\infty)}$ has compact support. Define

$$
w\left(x_{0}, t\right)=\int_{R^{n}}\left(f(x)+\int_{0}^{t} g(x, \tau)-u^{(\infty)}(x, t)\right) \Gamma_{x_{0}} d x
$$

where $\Gamma_{x_{0}}$ is the fundamental solution of the Laplacian with singularity at $x_{0}$. Note that $w\left(x_{0}, t\right)$ is well defined since $u^{(\infty)}$ has compact support. It is clear that

$$
-\Delta w=f(x)+\int_{0}^{t} g(x, \tau) d \tau-1
$$

in $N_{t}$ (since $u^{(\infty)}(x, t)=1$ on $\left.N_{t}\right)$.

On the other hand,

$$
\begin{aligned}
w^{(m)}\left(x_{0}, t\right) & =\int_{R^{n}}\left[u^{(m)}(x, t)-f(x)-\int_{0}^{t} g(x, \tau) d \tau\right] \Gamma_{x_{0}} d x \\
& =\int_{0}^{t} \int_{R^{n}} \Delta\left(u^{(m)}\right)^{m} \Gamma_{x_{0}} d x d \tau \\
& =\int_{0}^{t}\left(u^{(m)}\right)^{m}\left(x_{0}, \tau\right) d \tau
\end{aligned}
$$

Clearly, $w^{(m)}\left(x_{0}, t\right) \geq 0$ for any $x_{0} \in R^{n}$ and $t \in[0, T]$. By Step 4, we see that if $x_{0} \in S_{t}$, then there exists a small ball $B_{\delta^{\prime}}\left(x_{0}\right) \subset S_{t}$. This implies

$$
u^{\left(m^{\prime}\right)}\left(x_{0}, \tau\right) \leq \theta<1
$$

for all $\tau \in[0, t]$, provided that $m^{\prime}$ is sufficiently large. Consequently, $w^{(m)}\left(x_{0}, t\right) \rightarrow 0$ as $m \rightarrow \infty$. Thus,

$$
\begin{aligned}
w(x, t) \geq 0, & \text { for a.e. } x \in R^{n}, t \in[0, T], \\
w\left(x_{0}, t\right)=0, & \text { if } x_{0} \in N_{t},
\end{aligned}
$$

i.e., $w(x, t)$ is a solution of the variational inequality (4.3)-(4.5). 
5. The profile of the current density for limit solution. Throughout this section we assume that

$$
\mathbf{H}=\left\{h_{1}, h_{2}, 0\right\} .
$$

We shall use the results from the previous section to derive the profile of the current density.

$$
\text { Assume that } L=\left\|\nabla \times \mathbf{H}_{0}\right\|_{L^{\infty}\left(R^{2}\right)}>1 \text {. }
$$

Assume that $\mathbf{F}$ and $\mathbf{H}_{0}$ satisfy the assumptions $\mathrm{H}(4.1)-\mathrm{H}(4.2)$, where $u_{0 i}$ and $g_{i}$ are defined as in Sec. 2 .

First of all, since

$$
\nabla \mathbf{H}_{0}=h_{01 x_{1}}+h_{02 x_{2}}=0
$$

we see by the definition that

$$
u_{01}(x)=u_{02}(x)=h_{02 x_{2}}(x)-h_{01 x_{1}}(x) .
$$

Similarly, since $\nabla \times F=0$ one can find that

$$
g_{1}(x, t)=g_{2}(x, t), \quad \text { in } Q_{T} .
$$

Define the current densities $J_{p}$ and $J$ as follows:

$$
J_{p}(x, t)=\left|\nabla \times \mathbf{H}^{(p)}\right|, \quad J(x, t)=\left|\nabla \times \mathbf{H}^{(\infty)}\right| .
$$

Note that $J$ is well defined since $\nabla \times \mathbf{H}^{(\infty)}$ is well defined.

TheOREM 5.1. Under the assumptions $\mathrm{H}(5.1)-\mathrm{H}(5.2)$, the current density $J(x, 0+)=1$ if $x \in N_{0}$ and $J(x, 0+)=\left|\nabla \times \mathbf{H}_{0}\right|$ if $x \in S_{0}$, where $N_{0}=R^{2} \backslash S_{0}$, and $S_{0}$ is the noncoincident set of a variational inequality

$$
-\Delta w \geq g-1, \quad w \geq 0, \quad\left(\Delta w+g_{i}-1\right) w=0 .
$$

Proof. From Lemma 4.3, we know that

$$
u_{i}^{(\infty)}(x, 0+)=v^{(\infty)}(x) .
$$

On the other hand, we know that

$$
u_{i}^{(p)}=\Delta U^{(p)}=\left|\nabla \times \mathbf{H}^{(p)}\right| .
$$

It follows that

$$
J_{p}(x, t) \rightarrow J(x)
$$

as $p \rightarrow+\infty$. Thus, in the superconductor region, denoted by $S_{0}$, the current density

$$
J(x, 0+)=v^{(\infty)}(x)=\left|\nabla \times \mathbf{H}_{0}\right|, \quad x \in R^{2} .
$$

In the normal conductor region, denoted by $N_{0}$, the current density

$$
J(x, 0+)=v^{(\infty)}(x)=1 .
$$

Moreover, $S_{0}$ is the noncoincident set of a variational inequality (see [5]):

$$
-\Delta w \geq u_{0 i}-1, \quad w \geq 0, \quad\left(\Delta w+u_{0 i}-1\right) w=0 .
$$


Next we assume that

$$
\left\|\nabla \times \mathbf{H}_{0}\right\|_{L^{\infty}\left(R^{2}\right)} \leq 1
$$

Assume that $\mathbf{H}_{0}$ and $\mathbf{F}$ satisfy the assumptions $\mathrm{H}(4.3)$, where $u_{0 i}$ and $g_{0 i}$ are defined the same as in Sec. 2.

TheOREm 5.2. Under the conditions $\mathrm{H}(5.1)-\mathrm{H}(5.2)^{\prime}$ the current density $J(x, t)=\mid \nabla \times$ $\mathbf{H}^{(x)} \mid$ has the following structure:

$$
J(x, t)=\left\{\begin{array}{l}
1, \quad \text { if } x \in N_{t}, t>0, \\
f(x)+\int_{0}^{t} g(x, \tau) d \tau, \quad \text { if } x \in S_{t}, t>0 .
\end{array}\right.
$$

where $N_{t}=R^{2} \backslash S_{t}$ and $S_{t}$ is the noncoincident set of the variational inequality

$$
\begin{gathered}
w(x, t) \geq 0, \quad-\Delta w \geq u_{0 i}+\int_{0}^{t} g_{i} d \tau-1, \\
w\left(\Delta w+u_{0 i}+\int_{0}^{t} g_{i} d \tau-1\right)=0 .
\end{gathered}
$$

Proof. From Theorem 4.8, we see that

$$
J(x, t)=u_{i}^{(x)}(x, t) .
$$

The rest of the proof follows the same procedure as that of Theorem 5.1.

Next we show that the limit solution of (1.1)-(1.3) satisfies a degenerate evolution system.

THEOREM 5.3. There exists a nonnegative, bounded, and measurable function $a(x, t)$ such that the limit solution $\mathbf{H}^{(x)}$ satisfies

$$
\mathbf{H}_{t}^{(x)}-\nabla \times\left[a(x, t) \nabla \times \mathbf{H}^{(x)}\right]=\mathbf{F}, \quad(x, t) \in Q_{T},
$$

in the sense of distribution. Moreover,

$$
\operatorname{supp} a \subset N_{t} \quad \text { for a.e. every } t \in[0, T] .
$$

Proof. The proof is similar to [9]. Define

$$
\mathbf{A}_{p}=|\nabla \times \mathbf{H}|^{p-2} \nabla \mathbf{H}
$$

Then, for any $\mathbf{V} \in H\left(0, T ; B^{d}\right)$ with $\operatorname{supp} \mathbf{V} \in B_{R}(0)$ for each $t \in(0, T]$, we have

$$
\begin{gathered}
\int_{0}^{T} \int_{B_{R}(0)}\left[-\mathbf{H}^{(p)} \cdot \mathbf{V}_{t}+\mathbf{A}_{p} \cdot \nabla \mathbf{V}\right] d x d t \\
=\int_{0}^{T} \int_{B_{R}(0)} \mathbf{F} \cdot \mathbf{V} d x d t
\end{gathered}
$$

By Lemma 4.6, we know that there exists a subsequence such that

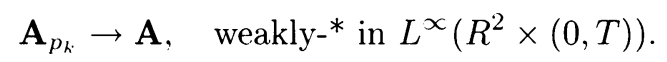


It follows that

$$
\begin{gathered}
\int_{0}^{T} \int_{B_{R}(0)}\left[-\mathbf{H}^{(\infty)} \cdot \mathbf{V}_{t}+\mathbf{A} \cdot \nabla \times \mathbf{V}\right] d x d t \\
=\int_{0}^{T} \int_{B_{R}(0)} \mathbf{F} \cdot \mathbf{V} d x d t
\end{gathered}
$$

On the other hand, we take $\mathbf{V}=\mathbf{H}^{(p)}$ as a test vector to obtain

$$
\begin{aligned}
& \frac{1}{2} \int_{B_{R}(0)}\left|\mathbf{H}^{(p)}\right|^{2} d x+\int_{0}^{T} \int_{B_{R}(0)}\left|\nabla \mathbf{H}^{(p)}\right|^{p} d x d t \\
& \quad=\int_{0}^{T} \int_{B_{R}(0)} \mathbf{F} \cdot\left(\mathbf{H}^{(p)}\right) d x d t \rightarrow \int_{0}^{T} \int_{B_{R}(0)} \mathbf{F} \cdot\left(\mathbf{H}^{(\infty)}\right) d x d t .
\end{aligned}
$$

It follows that

$$
\int_{0}^{T} \int_{B_{R}(0)}\left|\nabla \times \mathbf{H}^{(p)}\right|^{p} d x d t \rightarrow \int_{0}^{T} \int_{B_{R}(0)} \mathbf{A} \cdot(\nabla \times \mathbf{H}) d x d t
$$

as $p \rightarrow \infty$.

By Fatou's lemma, we have

$$
\begin{aligned}
& \int_{0}^{T} \int_{B_{R}(0)}|\mathbf{A}| d x d t \leq \liminf _{p_{k} \rightarrow \infty} \int_{0}^{T} \int_{B_{R}(0)}\left|\mathbf{A}_{p_{k}}\right| d x d t \\
& \quad \leq \lim _{p_{k} \rightarrow \infty}\left(\int_{0}^{T} \int_{B_{R}(0)}\left|\nabla \times \mathbf{H}^{\left(p_{k}\right)}\right|^{p_{k}} d x d t\right)^{1-\frac{1}{p_{k}}}\left[T\left|B_{R}(0)\right|\right]^{\frac{1}{p_{k}}} \\
& \quad=\int_{0}^{T} \int_{B_{R}(0)} \mathbf{A} \cdot\left(\nabla \times \mathbf{H}^{(\infty)}\right) d x d t .
\end{aligned}
$$

It follows that

$$
|\mathbf{A}|=\mathbf{A} \cdot\left(\nabla \times \mathbf{H}^{(\infty)}\right),
$$

since $\left|\nabla \times \mathbf{H}^{(\infty)}\right| \leq 1$. Consequently, there exists a nonnegative, bounded function $a(x, t)$ such that

$$
\mathbf{A}(x, t)=a(x, t) \nabla \times \mathbf{H}^{(x)} .
$$

Moreover, if $\nabla \times \mathbf{H}^{(x)}$ exists and $\left|\nabla \times \mathbf{H}^{(x)}\right|<1$, then $a(x, t)=0$.

Acknowledgment. The author would like to thank Professor L. C. Evans and Dr. M. Feldman for many helpful discussions during the preparation of this paper.

This work was done at MSRI, University of California, Berkeley, CA 94720. The author would also like to thank the Directors and Staff at MSPI for providing an excellent research environment. 


\section{REFERENCES}

[1] G. Aronsson, L. C. Evans, and Y. Wu, Fast/slow diffusion and growing sandpiles, J. Differential Equations 131, 304-335 (1996)

[2] A. Bossavit, Numerical modeling of superconductors in three dimensions, a model and a finite element method, IEEE Trans. Magn. 30, 3363-3366 (1994)

[3] C. P. Bean, Magnetization of high-field superconductors, Rev. Mod. Phys. 36, 31-39 (1964)

[4] Ph. Benilan and M. Crandall, The continuous dependence on $\phi$ of solutions of $u_{t}-\Delta \phi(u)=0$, Indiana Univ. Math. J. 30, 161-177 (1981)

[5] L. A. Caffarelli and A. Friedman, Asymptotic behavior of solutions of $u_{t}=\Delta u^{m}$ as $m \rightarrow \infty$, Indiana Univ. Math. J. 36, 711-728 (1987)

[6] E. DiBenedetto, Degenerate Parabolic Equations, Springer-Verlag, New York, 1993

[7] E. DiBenedetto, Continuity of weak solutions to a general porous medium equation, Indiana University Math. J. 32, 83-118 (1983)

[8] L. C. Evans, M. Feldman, and R. F. Gariepy, Fast/slow diffusion and collapsing sandpiles, J. Differential Equations 37, 166-209 (1997)

[9] L. C. Evans and W. Gangbo, Differential equations methods for the Monge-Kantorovich mass transfer problem, Mem. Amer. Math. Soc. 137 (1999)

[10] A. Friedman, Variational Principles and Free Boundary Problems, John Wiley and Sons, New York, 1982

[11] D. Gilbarg and N. Trudinger, Elliptic Partial Differential Equations of Second Order, SpringerVerlag, Berlin, 2nd ed., 1983

[12] L. D. Landau and E. M. Lifshitz, Electrodynamics of Continuous Media, Pergamon Press, New York, 1960

[13] O. A. Ladyzenskaja, V. A. Solonnikov, and N. N. Ural'ceva, Linear and Quasilinear Equations of Parabolic Type, Translations of Mathematical Monographs, Vol. 23, Amer. Math. Soc., Providence, RI, 1967

[14] L. Prigozhin, Variational model of sandpile growth, European J. Appl. Math. 7, 225-235 (1996)

[15] L. Prigozhin, Solution of thin film magnetization problems in type-II superconductivity, J. Comput. Phys. 144, 180 193 (1998)

[16] M. Struwe, Variational Methods, Springer-Verlag, Berlin, 1990

[17] H. M. Yin, Regularity of solutions to Maxwell's system in quasi-stationary electromagnetic fields and applications, Comm. Partial Differential Equations 22, 1029-1053 (1997) 